\title{
Globalbudget: Mit der Tarnkappe durchs Parlament
}

\section{Yvonne Gilli}

Dr. med., Präsidentin der FMH

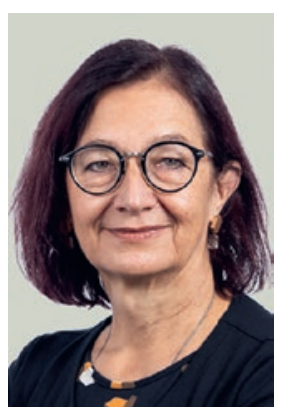

Wenn es um die Kostendämpfung im Gesundheitswesen geht, finden derzeit vor allem zwei Vorhaben Aufmerksamkeit: Die im März 2020 eingereichte Kostenbremse-Initiative der Mitte-Partei sowie die sogenannte «Zielvorgabe», die der Bundesrat am 10. November als indirekten Gegenvorschlag dazu vorgelegt hat. Beide Vorhaben fordern einen Kostendeckel für die Gesundheitsversorgung, gehen dessen Ausgestaltung jedoch sehr verschieden an.

Die Kostenbremse-Initiative fordert "wirksame Anreize», damit sich die Gesundheitskosten "entsprechend der schweizerischen Gesamtwirtschaft und den durchschnittlichen Löhnen» entwickeln [1]. Sollten zwei Jahre nach einer Annahme der Initiative keine verbindlichen Massnahmen festgelegt sein, die Kosten aber 20\% stärker als die Löhne wachsen, müsste «der Bund in Zusammenarbeit mit den Kantonen Massnahmen zur Kostensenkung» ergreifen [1]. Welche Massnahmen dies genau wären, bleibt völlig offen. Mehr Raum für die Ausgestaltung hätte die Mitte-Initiative gar nicht lassen können.

Insofern erstaunt die Begründung des Bundesrats für seinen Gegenvorschlag: «Der von der Initiative vorgeschlagene Mechanismus [sei] zu starr», so dass «je nach Umsetzung eine Rationierung der Leistungen» drohe [2]. Gerade der Gegenvorschlag sieht jedoch eine extrem engmaschige Regulierung vor: Mit der «Vorgabe von Zielen für die maximale Kostenentwicklung» sollen zukünftig staatlich definierte Kostenobergrenzen - differenziert nach «Kostenblöcken» und Kantonen - die Gesundheitsversorgung beschränken. Prämienzahlende dürften nicht mehr über die politischen «Kostenziele» hinaus behandelt werden, wenn Leistungserbringer staatliche Tarifeingriffe oder andere Sanktionen vermeiden möchten. Damit wäre aus Sicht der FMH erst recht eine «Rationierung von Leistungen» [3] zu befürchten. Zudem würden mit «aufwändiger und detaillierter Planwirtschaft» des Bundes im Gesundheitswesen keine «echten Lösungen» gefunden, wie die Mitte-Partei kritisiert [4].

Bei allen Unterschieden bleibt beiden Vorlagen gemeinsam, dass sie unserem Gesundheitswesen ein Globalbudget auferlegen möchten und damit einen gesundheitspolitischen Grundsatzentscheid erfordern. Aktuell deutet jedoch vieles darauf hin, dass eine solche Grundsatzdiskussion gar nicht geführt werden soll: Der Systemwechsel hin zu einer Budgetierung mit einschneidenden Folgen für unsere Patientenversorgung könnte bereits in dieser Wintersession vorweggenommen werden. Denn während «Kostenbremse» und «Zielvorgaben» die Aufmerksamkeit auf sich ziehen, steht der dem Parlament vom EDI vorgelegte neue Artikel 47c KVG kurz vor der Verabschiedung im Ständerat. Auch dieser Artikel fordert eine Kostenobergrenze im Sinne der bundesrätlichen «Zielvorgabe»: Die Tarifpartner sollen künftig gemäss politischen Vorgaben die «gerechtfer-

\section{Während "Kostenbremse» und "Zielvorgaben»} die Aufmerksamkeit auf sich ziehen, steht der Artikel 47c KVG kurz vor der Verabschiedung.

tigten» Kosten eines Kalenderjahres festlegen. Überschreiten sie dieses vorab fixierte Globalbudget, drohen finanzielle Sanktionen. Auch hier würde also der Versicherungsanspruch der Patientinnen und Patienten durch ein Budget beschränkt - und Ärztinnen und Ärzte dazu angehalten, im Zweifelsfall Leistungen vorzuenthalten, wenn sie finanzielle Sanktionen vermeiden möchten. Der Bundesrat hielt sogar explizit zum Artikel 47c fest, dieser solle «dazu dienen, diese Zielvorgabe zu erreichen» [5]. Bevor also der bundesrätliche Gegenvorschlag überhaupt abgestimmt wird, würden hier bereits Umsetzungsdetails vorweggenommen.

Es bleibt zu hoffen, dass die kleine Kammer der Tragweite des Artikels 47c Rechnung trägt - und entscheidet, ihn erst gemeinsam mit der Kostenbremse-Initiative und dem bundesrätlichen Gegenvorschlag zu beraten. Andernfalls wäre die Einführung eines Kostendeckels in der Gesundheitsversorgung bereits beschlossen, bevor die grossen Vorlagen dazu überhaupt diskutiert worden sind. Ein solch folgenreicher Eingriff in ein so erfolgreiches und geschätztes Gesundheitswesen verdient jedoch mehr Aufmerksamkeit.

\section{Literatur}

Die vollständige Literaturliste finden Sie in der Online-Version des Artikels unter www.saez.ch 